\title{
The Ultrasonic Sensor System Based on the Chip of SH79F32
}

\author{
Wenkai Zhang ${ }^{1, a}$
}

${ }^{1}$ School of science, Beijing Information Science and Technology University, Beijing100192, China

aemail:bjxxkjdx408@sina.com,

Keywords: Ultrasonic sensorss, HC-SR04, SH79F32, Measurement

\begin{abstract}
In this thesis, we take SH79F32 as main chip, LCD12864 as the display screen,the ultrasonic sensor of HC-SR04 as collection tools and reach the function of ultrasonic distance measurement. Through Altium Designer Release 9 to conduct schematic design and draw PCB, and then solder circuit boards, use the software of Keil to design process, to realize ultrasonic sensor measurement, after that ,complete the design and production of ultrasonic sensor design and production.
\end{abstract}

\section{.Introduction}

The Ultrasonic Sensor System has the function of data acquisition and processing, which can be monitored, and the object of the sensor and the monitoring. Because it is in the distance, with relatively fast, convenient, simple calculation, easy real-time control, high accuracy, high reliability, and other characteristics. Suitable for high temperature, high dust, high humidity, strong electromagnetic interference and other adverse environment. Therefore, it has a broad application prospect. The design of wireless ultrasonic sensor platform system.

\section{The design of ultrasonic sensor system}

Working of the ultrasonic sensor system shown in the Fig. 1 It is SH79F32 chip, and it externally connects LCD12864, digital ultrasonic sensor HC-SR04 and so on.

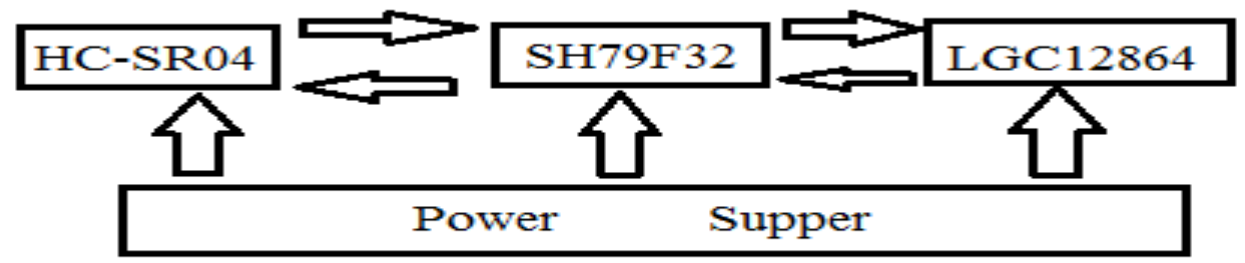

SH7F932

Fig.1 Working principle of the ultrasonic sensor

In this thesis, the control circuit module is designed with SH79F32 chip as the core, SH79F32 is a low power microcontroller with high speed clock and storage period 8051. At the same frequency of oscillation, it is better to run faster than the traditional 8051.

In addition to the built-in SH79F32 256 bytes of RAM and 16 three bit timer / counters, a UART, external interrupt INT0 and INT1, and IR, TWI, EUART, SPI and other standard communication module and LCD driver, 512 bytes of external RAM, built-in functions are compared to ADC, PWM timer and real-time clock module. In order to achieve high reliability and low power consumption ${ }^{[1]}$, SH79F32 PLL clock, SLP (ultra low power) LCD driver, watchdog timer, low voltage reset function, low voltage detection function. In addition there are two SH79F32 low power saving mode.

\section{LCD12864}

Display module of the system is not complicated characters to be displayed, LCD12864 meet the design requirements. 1602LCD is an industrial character LCD with Chinese character; interior includes GB one, two simplified Chinese character dot-matrix graphic LCD module; its display 
resolution of $128 \times 64$, built $819216 * 16: 00$ Chinese characters, and $12816 *$ 8:00 ASCII character set. The module is flexible and simple interface mode, there are 4/8 bit parallel, two-wire or three-wire serial interface to a variety of ways, which can simultaneously display 16x02 32 characters. Usually have 14 pin cable or 16 pin cable ${ }^{[2]}$, the extra two lines is the backlight power cord. LCD12864 pin shown in Figure 2.

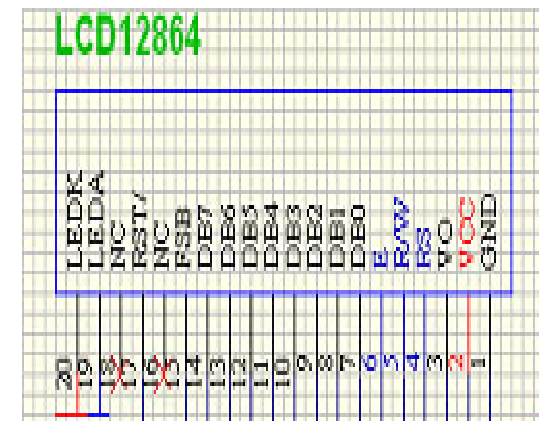

Fig.2 LCD12864 pin arrangement

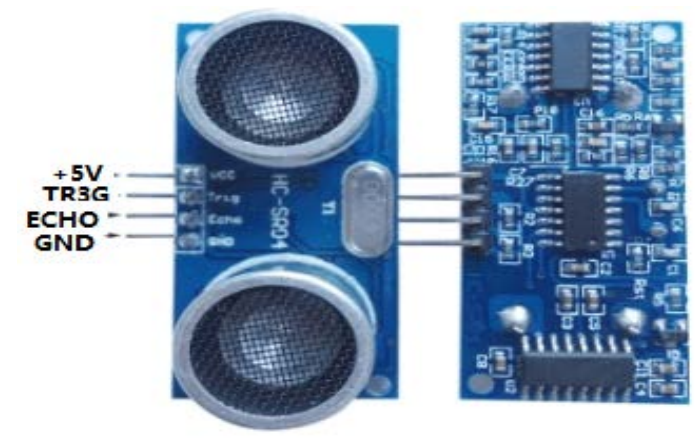

Fig.3 HC-SR04

\section{HC-SR04}

The Ultrasonic sensor adopts HC-SR04, which can provide $2 \mathrm{~cm}-400 \mathrm{~cm}$ non-contact distance sensing capabilities, ranging accuracy can reach high to $3 \mathrm{~mm}$; module comprises an ultrasonic transmitter, receiver and control circuitry. The basic working principle is as follows.

The Ultrasonic sensor adopts HC-SR04, which can provide $2 \mathrm{~cm}-400 \mathrm{~cm}$ non-contact distance sensing capabilities, ranging accuracy can reach high to $3 \mathrm{~mm}$; module comprises an ultrasonic transmitter, receiver and control circuitry. The basic working principle is as follows.

(1) using the IO port TRIG trigger ranging, high signal to at least 10us.

(2) the module automatically sends eight $40 \mathrm{khz}$ square wave, automatically detects whether a signal return;

(3) a signal back, through the IO port ECHO outputs a high level, high duration is Ultrasonic time from launch to return. Test distance $=($ time $*$ High speed of sound $(340 \mathrm{M} / \mathrm{S})) / 2$;

HC-SR04 ultrasonic ranging module wiring (Figure 3) shows: VCC 5V power supply, GND is ground, TRIG trigger control signal input, ECHO echo signal output in four interface ports ${ }^{[3]}$.

\section{Hardware design of ultrasonic sensor system}

The hardware design of ultrasonic wave is made up of five parts, which are power circuit design, control circuit design, ultrasonic ranging circuit and display circuit design;

\section{Power supply circuit}

Because SH79F32 and LCD12864 need 5v voltage, while the wireless transmitter module requires $3.3 \mathrm{v}$ power supply, therefore, the design used $9 \mathrm{v}$ battery as a power supply circuit, so the power supply circuit shown in Fig.4.

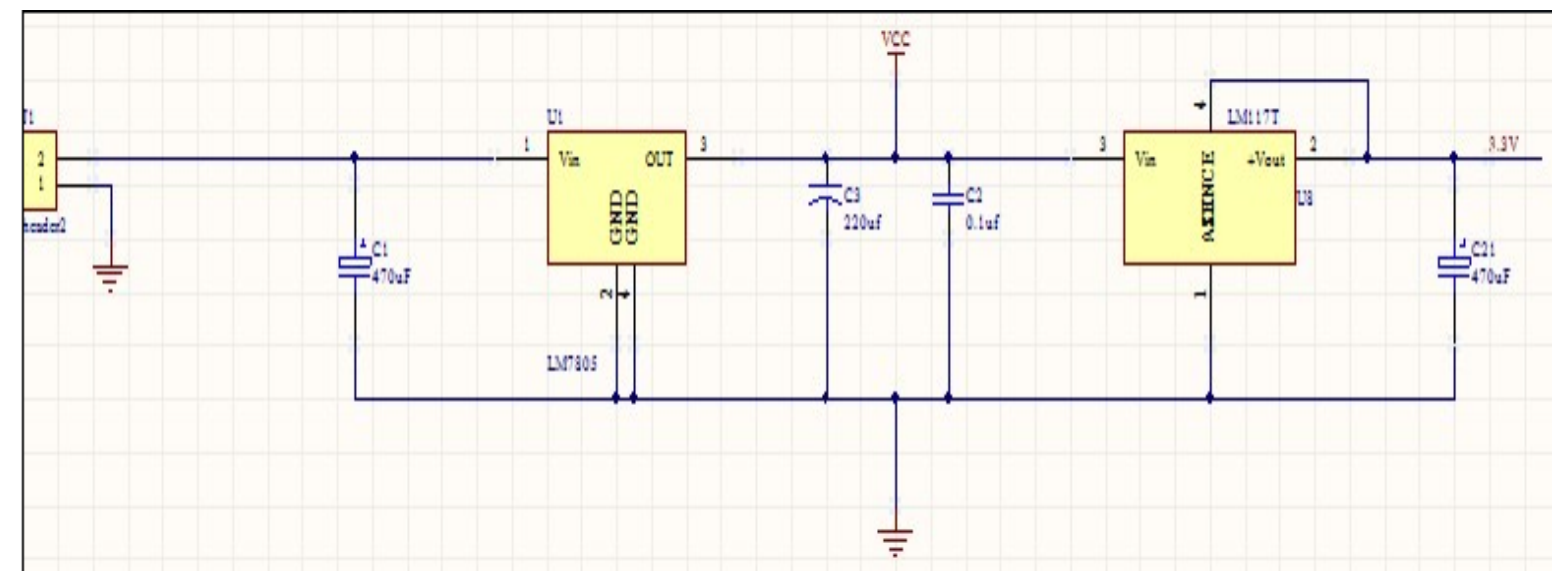

Fig.4 power supply circuit 


\section{LCD circuit AND HC-SR04 circuit}

The ultrasonic sensor system, LCD module ${ }^{[4]}$ (Figure 5), the wireless transmitter module, power modules are the same. From the machine design an HC-SR04 Ultrasonic Ranging Module interface circuit (Figure 6).

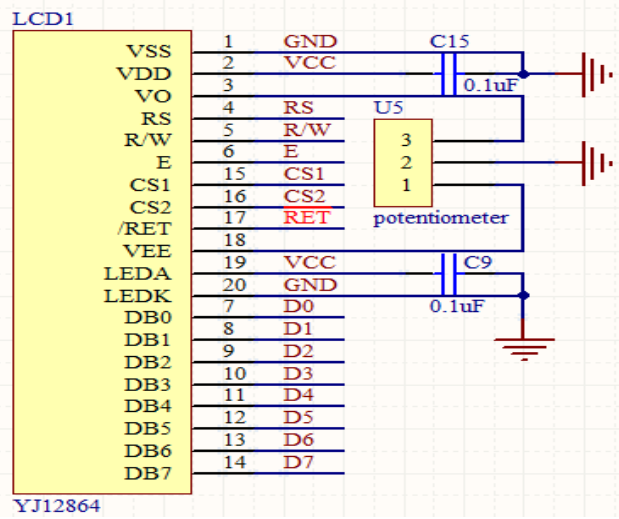

Fig.5 LCD12864 Circuit

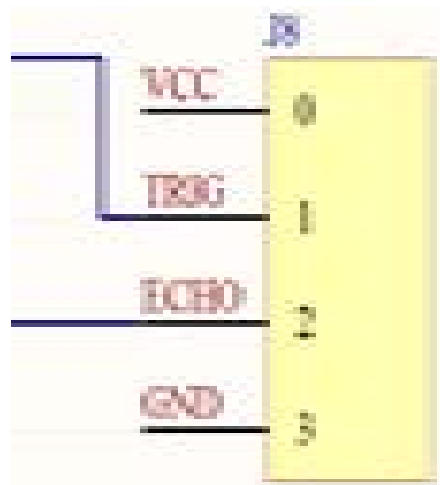

Fig.6 HC-SR04 Interface Circuit

\section{Power supply circuit}

Because SH79F32 and LCD12864 need 5v voltage, while the wireless transmitter module requires $3.3 \mathrm{v}$ power supply, therefore, the design used $9 \mathrm{v}$ battery as a power supply circuit ${ }^{[5]}$.

\section{The design of the SH79F32 Control circuit}

In SH79F32 microcontroller as the core control circuit (Figure 7), the figure J4 is burned into the chip program to interface through the TCK, TMS, TDO, TDI four pin poured into programming mode specific sequence . P0 port 8 data lines connected to the LCD module data port, P1.2 P1.7 control port connected to the LCD module, P4 port, P2.6, P2.7, P3.7, and wireless module interface is connected, P3. two and drawn two lines belong to P3.3 serial communication line, through J8 conversion interface, and HC-SR04 ultrasonic ranging module. Control HC-SR04 ultrasonic ranging module.

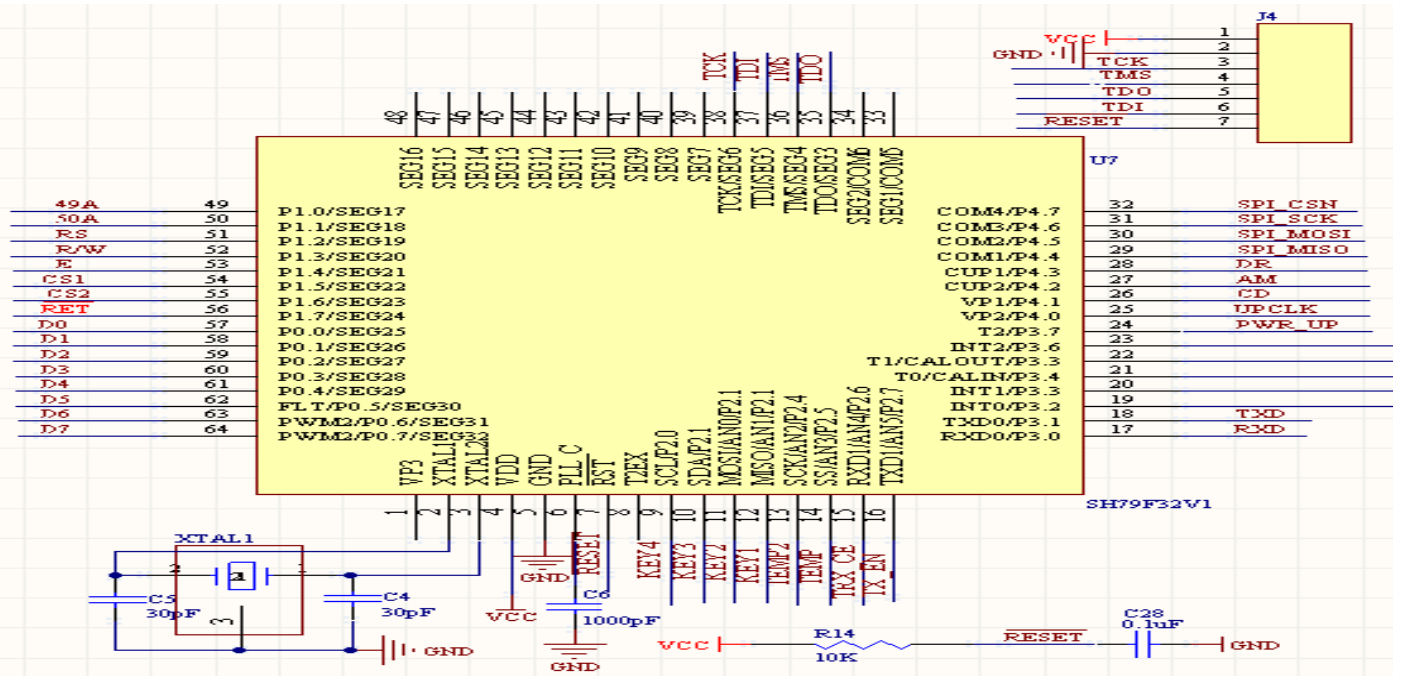

Fig.7 SH79F32 control circuit

\section{Performance test}

On 9V battery, the LCD screen displays START, press the switch K1 to display S, then start ranging, such as figure 8, press switch K2, stop ranging, the screen displays $\mathrm{C}$, end of the test. 


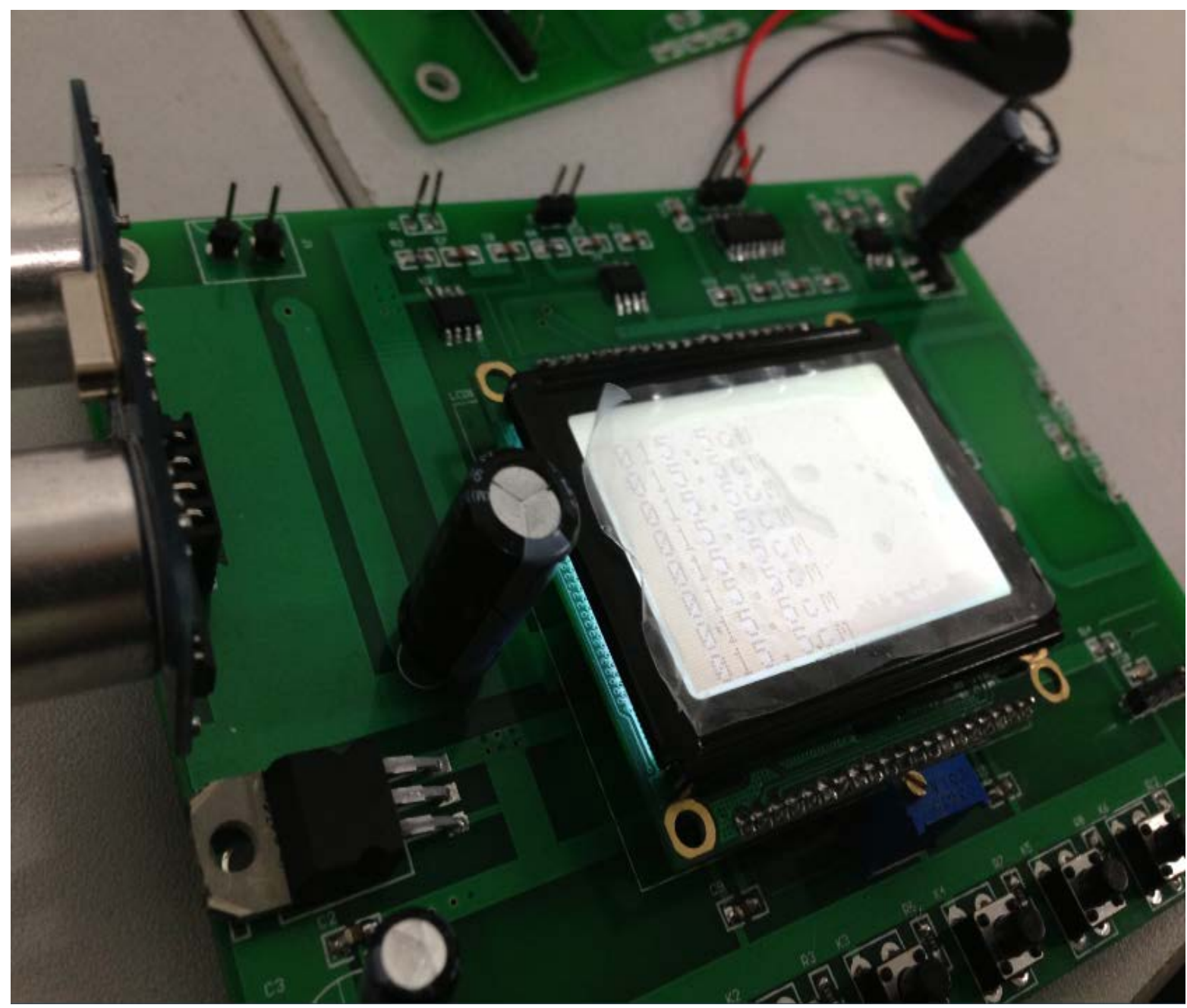

Fig.8

Test results

\section{Acknowledgment}

This work was supported by the Practice base construction project of the Beijing Information \& Technology University No.5111523908.

\section{References}

[1] Information on http://www.sinowealth.com

[2] Information on http:// wenku.baidu.com

[3] Information on http:// xycsensor.cn.alibaba.com

[4] Min Shen, Binwu Wang,: LCM045 module with MCU Interface Design [J], Journal of Guilin College of Aerospace Technology, 2007.

[5] Lixia Li : SCM Ultrgisonic Ranging Applications [J], Electronic technology, 2002(6). 